\title{
Combining Wireless Technology and Behavioral Economics to Engage Patients (WiBEEP) with cardiometabolic disease: a pilot study
}

Edith Angellotti ${ }^{*}$ D, John B. Wong ${ }^{2}$, Ayal Pierce ${ }^{3}$, Benjamin Hescott ${ }^{4}$ and Anastassios G. Pittas ${ }^{1}$

\begin{abstract}
Background: The long-term management of cardiometabolic diseases, such as type 2 diabetes and hypertension, is complex and can be facilitated by supporting patient-directed behavioral changes. The concurrent application of wireless technology and personalized text messages (PTMs) based on behavioral economics in managing cardiometabolic diseases, although promising, has not been studied. The aim of this pilot study was to evaluate the feasibility and acceptability of the concurrent application of wireless home blood pressure (BP) monitoring (as an example of "automated hovering") and PTMs (as an example of "nudging") targeting pharmacotherapy and lifestyle habits in patients with cardiometabolic disease (type 2 diabetes and/or hypertension).

Methods: The Wireless Technology and Behavioral Economics to Engage Patients (WiBEEP) with cardiometabolic disease study was a single-arm, open-label, 7-week-long pilot study in 12 patients (mean age 58.5 years) with access to a mobile phone. The study took place at Tufts Medical Center (Boston, MA) between March and September 2017. All patients received PTMs; nine patients received wireless home BP monitoring. At baseline, patients completed questionnaires to learn about their health goals and to assess medication adherence; at the end of week 7, all patients completed questionnaires to evaluate the feasibility and acceptability of the intervention and assess medication adherence. Hemoglobin A1c was ascertained from data collected during routine clinical care in 7 patients with available data.
\end{abstract}

Results: The majority of patients reported the text messages to be easy to understand (88\%) and appropriate in frequency (71\%) and language (88\%). All patients reported BP monitoring to be useful. Mean arterial pressure was lower at the end-of-study compared to baseline $(-3.4 \mathrm{mmHg}[95 \% \mathrm{Cl},-5$ to -1.8$]$. Mean change in hemoglobin A1c was $-0.31 \%[95 \% \mathrm{Cl},-0.56$ to -0.06$]$.

Conclusions: Among patients with cardiometabolic disease, the combination of wireless BP monitoring and lifestylefocused text messaging was feasible and acceptable. Larger studies will determine the long-term effectiveness of such an approach.

Keywords: Text messages, Blood pressure, Diabetes, Diet, Exercise

\footnotetext{
* Correspondence: EAngellotti@tuftsmedicalcenter.org

'Division of Endocrinology, Diabetes and Metabolism, Tufts Medical Center,

800 Washington Street, \#268, Boston, MA 02111, USA

Full list of author information is available at the end of the article
}

(c) The Author(s). 2019 Open Access This article is distributed under the terms of the Creative Commons Attribution 4.0 International License (http://creativecommons.org/licenses/by/4.0/), which permits unrestricted use, distribution, and reproduction in any medium, provided you give appropriate credit to the original author(s) and the source, provide a link to the Creative Commons license, and indicate if changes were made. The Creative Commons Public Domain Dedication waiver (http://creativecommons.org/publicdomain/zero/1.0/) applies to the data made available in this article, unless otherwise stated. 


\section{Background}

The rising prevalence of cardiometabolic diseases, such as type 2 diabetes and hypertension, challenges a U.S. healthcare system that was not designed for efficient management of chronic conditions. Effective long-term management of chronic diseases requires patients to adopt and sustain a healthy lifestyle in addition to taking prescribed medications. Patients with chronic diseases might spend only a few hours every year with a doctor or a nurse, but they spend about 5000 waking hours each year doing everything else-including deciding whether to take prescribed medications, deciding what to eat and drink, whether to smoke, whether to exercise and making other choices that can profoundly affect their health [1]. Despite the extensive availability of pharmacotherapies, nearly half of treated patients do not have adequate blood pressure (BP) or glycemic control $[2,3]$. The main contributors to not reaching therapeutic targets are poor medication adherence, lack of patient engagement and therapeutic inertia [4, 5]. Traditional medical care (including "routine" visits) accounts for only $\sim 10 \%$ of the total variance in outcomes for chronic conditions, while behavioral factors account for about 40\% [6]. Accordingly, there is a growing interest in developing simple methods to support patients outside the traditional healthcare settings to enhance the adoption of healthy lifestyle and medication adherence.

Over the past 10 years, the growth in wireless connectivity, the increased recognition for individualized medicine and the need for disruptive innovation to optimize health care value supported the development of the mobile health (mHealth)-defined as medical and public health practice supported by mobile (wireless) devices. The utilization of mHealth technologies is a promising approach to transform care of chronic conditions and support patients outside the traditional healthcare settings [7, 8]. Adherence to such technologies may be enhanced by applying principles from behavioral economics, defined as a combination of conventional economic principles with psychology to explain human behavior. Two behavioral economic principles of particular relevance to mHealth interventions include "automated hovering" and "nudging." The "automated hovering" (e.g., using wireless technology to monitor BP between clinic visits) can effectively fill the gap of those 5000 hours each year that patients spend away from the traditional health care setting. "Nudging" is the systematic and evidence-based development and implementation of nudges in creating behavior change (e.g., via text messages to influence predictable flaws in behavior) [1]. Behavioral economics to facilitate behavior change has also garnered substantial interest in healthcare delivery redesign but remains under-studied in type 2 diabetes [9-14]. The concurrent application of wireless technology and behavioral economic principles did not significantly improve medication adherence or vascular readmission outcomes after acute myocardial infarction [15], but has not been studied in chronic conditions such as type 2 diabetes and/or hypertension. The aim of this pilot study was to evaluate the feasibility and acceptability of combing wireless BP home monitoring (as an example of "automated hovering") and text messaging (as an example of "nudging") in patients with type 2 diabetes and/or hypertension.

\section{Methods \\ Study design}

The Wireless Technology and Behavioral Economics to Engage Patients (WiBEEP) with cardiometabolic disease study was a single-arm, open-label, 7-week-long pilot study. Its aim was to evaluate a strategy that included text messaging and wireless BP home monitoring targeting medication adherence and improved lifestyle habits (exercise and/or diet) in patients with cardiometabolic disease (type 2 diabetes and/or hypertension). All patients received automated personalized text messages (PTMs) and patients with hypertension also received wireless BP home monitoring. Participants attended only one in-person visit (baseline visit) plus two "virtual visits" (week 3 and week 7) if they qualified for wireless BP home monitoring. During the "virtual visits," patients received an email with mean blood pressure values and, if necessary, a suggestion for a change in antihypertensive therapy (previously discussed with the patient's primary care physician). During the baseline visit, once the informed consent form was signed, patients completed questionnaires to learn about their health goals (e.g., preventing stroke) and to assess medication adherence using the MMSA-8 Morisky scale [16]; patients and investigators jointly reviewed the data and collaboratively set goals for medication adherence, exercise, and diet.

\section{Participants and setting}

Patients were recruited from primary care and endocrinology clinics at Tufts Medical Center (Boston, MA): all patients who were pre-identified by their physician as potentially eligible were mailed a study invitation letter. When interested patients called back, a member of the research team verified the inclusion criteria and, if eligibility was confirmed, scheduled the baseline visit. Inclusion criteria were (a) established type 2 diabetes based on ICD codes (managed without or with pharmacotherapy) or hypertension based on ICD codes (managed without or with pharmacotherapy), (b) use of mobile phone able to receive and send texts (for all patients), and (c) use of a "smart" phone (for patients with hypertension). Exclusion criteria included (a) uncontrolled hypertension (systolic $\mathrm{BP}>180 \mathrm{mmHg}$ or diastolic $\mathrm{BP}>$ $120 \mathrm{mmHg}$ ), (b) pregnancy (past 1 year by report), (c) 
intent to become pregnant in the next year, or (d) any unstable medical or psychiatric condition or (e) any other reason that, in the opinion of the investigators, would impede adherence with study procedures or hinder completion of the study or increase risk. The study took place between March and September 2017. The Tufts IRB approved the study, and all participants provided written informed consent.

\section{Intervention \\ Nudging}

All patients received PTMs that were semi-customized with the content selected from a bank of short, actionable options providing patient-tailored advice and motivation towards healthy lifestyle changes (exercise and/or diet), and increased adherence to cardio-metabolic (i.e., diabetes, hypertension) medications. Prior studies have reported that interventions with personalized messages have a larger effect size [17]; therefore, certain elements of the texts were semi-personalized via a "mail-merge" function with the patient's preferred name, providing reminders, encouragement and motivation to improve adherence to medication, increase exercise or improve diet, based on patient's needs and preferences. The bank of messages was developed with input from investigators, and content was developed for 3 modules: exercise, diet, and medication adherence. Personalized text messages followed key insights from behavioral economics (e.g., limiting choice overload, setting goal gradients) and were personalized based on patient-specified personal health goals identified in the baseline questionnaire. Frequency (times per week) and timing (time of the day) of PTMs was determined by the patient. Some PTMs were merged with patient's preferred names. Some PTMs were bi-directional requesting patients to respond to a question with a simple "yes" or "no". Table 1 provides examples of messages.

\section{Automated hovering}

In addition to texting, 9 patients with hypertension managed without $(n=1)$ or with $(n=8)$ pharmacotherapy received wireless $\mathrm{BP}$ home monitoring using an FDA-approved device (iHealth Labs, Inc.) connected via Bluetooth to the patient's phone (or tablet). During the baseline visit, investigators demonstrated the use of the monitor to ensure that patients understood the procedure and provided written step-by-step instructions. Patients received a welcome email with a link to a login page to set up a unique username and password. This subgroup was asked to check BP measurements twice a day after 5 or more minutes of rest, comfortably seated in a quiet room with their feet touching the floor; results were automatically and wirelessly collected via the iHealth MyVitals App into a cloud-based portal
Table 1 Examples of text messages used in the WiBEEP pilot study Diet

At your next meal, and every day, fill half of your plate with fruits and vegetables.

At your next meal, stay away from processed (white) carbohydrates.

Choose fish high in omega-3 fatty acids, like salmon, trout, and herring.

Exercise

Take the stairs. Your body and your brain will thank you!

Do not forget to exercise today. Exercise lowers risk of stroke.

Today, round up a friend or family member and walk. Team sport is fun!

Medication adherence

Hello [NAME], remember to take your medicines today at [TIME].

Medications work best if taken at a particular time. Don't forget your medications today.

Use a pillbox with compartments for each day: it prevents double doses.

(sherbit.com) accessible to study staff. Acceptability of the intervention components (wireless BP monitoring and text messaging), improvements in medication adherence (MMSA-8 Morisky), and BP (collected via the monitors) were assessed at the end of the study. In 7 patients, change in HbA1c was also ascertained from available data in electronic health records collected during routine clinical care.

\section{Results}

In total, 35 patients were invited to participate in the pilot study. Of those, 13 were eligible and 12 completed the study (Fig. 1). Mean age of patients was 58.5 years (range 41 to 71 ), and BMI was $31 \mathrm{~kg} / \mathrm{m}^{2}$. Twenty-five percent did not have a college degree (Table 2).

All patients received PTMs with a total of 454 messages sent; 95 were bidirectional. Patients responded to $50 \%$ of the bidirectional texts. Most patients reported that PTMs were easy to understand (88\%), and appropriate in frequency $(71 \%)$ and language $(88 \%)$. We collected $77 \%$ of expected BP recordings. Nine participants with a history of hypertension received the wireless BP home monitoring. All of them reported the $\mathrm{BP}$ monitoring to be useful, and nearly all (89\%) found it easy to use. At the end of the study, $82 \%$ of participants reported high medication adherence vs. $75 \%$ at baseline. After review of $\mathrm{BP}$ results at the mid-point, a change in anti-hypertensive therapy was made in 4 out of 9 patients (3 changed prescriptions; 1 reduced salt intake). Mean BP improved from $147 / 84 \mathrm{mmHg}$ (week 0) to $143 / 81 \mathrm{mmHg}$ (week 4-7). Mean arterial pressure was lower at the end-of-study compared to baseline $(-3.4 \mathrm{mmHg}$ [95\% CI, -5 to -1.8 ]; Fig. 2a). In patients with available data $(n=7), \mathrm{HbA1c}$ was lower in most patients (Fig. 2b) compared to baseline. 


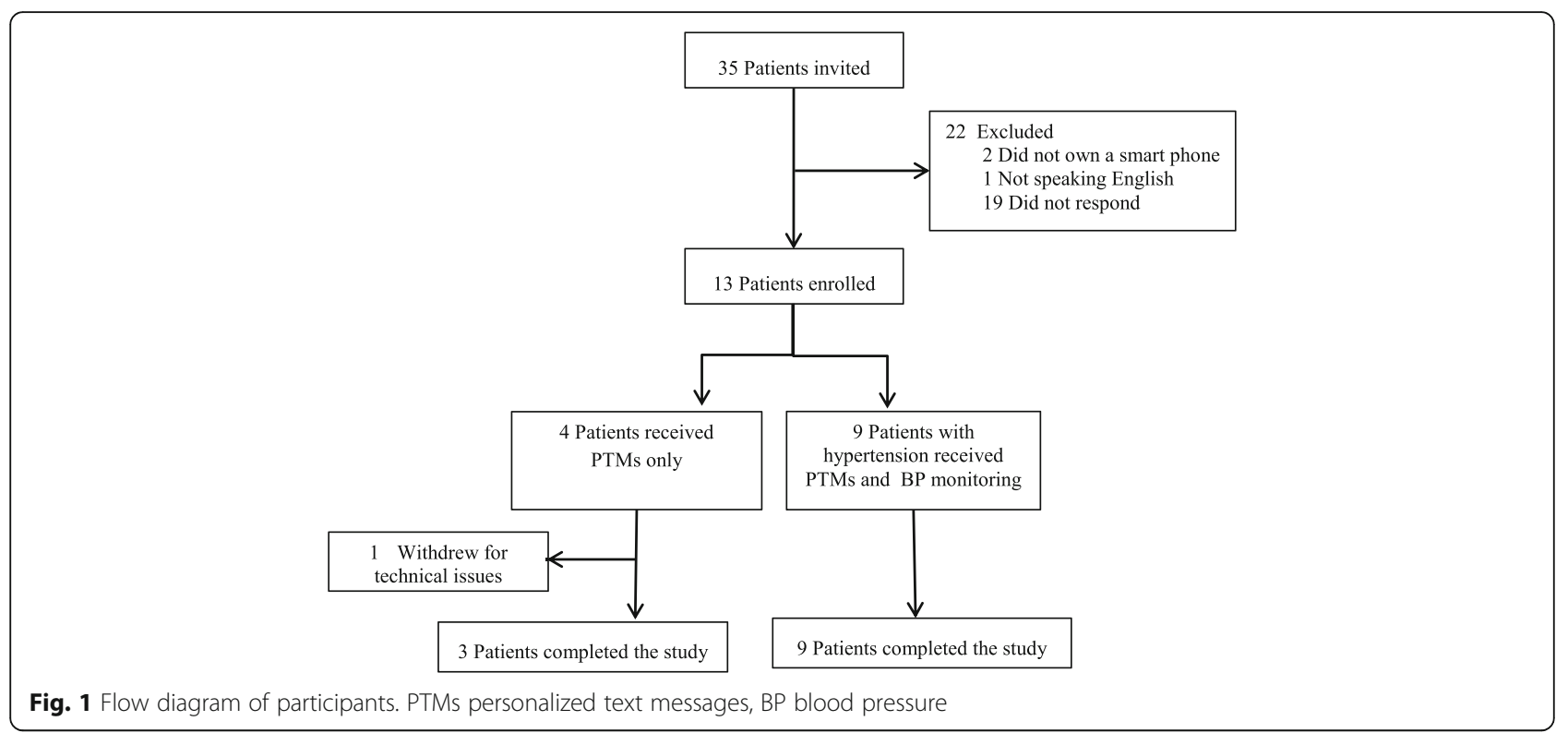

Mean change in HbA1c among all patients was $-0.31 \%$ [ $95 \% \mathrm{CI},-0.56$ to -0.06 ].

\section{Discussion}

This pilot study provided valuable data to improve and redefine study procedures (such as recruitment method,

Table 2 Baseline patient characteristics

\begin{tabular}{ll}
\hline Characteristics & $N=12$ \\
\hline Demographics & \\
Age, years & $58.5+/-8.5$ \\
Female, $n$ (\%) & $3(25)$ \\
Education, $n$ (\%) & \\
$\quad$ Bachelor degree or higher & $9(75)$ \\
$\quad$ Advanced diploma/diploma & $2(17)$ \\
$\quad$ School certificate & $1(8)$ \\
Employment, $n$ (\%) & \\
Full time employment & $8(67)$ \\
Employment part-time/casual & $1(8)$ \\
Retired/pensioner & $2(17)$ \\
Other & $1(8)$ \\
Current/former smoker, $n(\%)$ & $4(33)$ \\
Clinical characteristics & \\
Body mass index, kg/m ${ }^{2}$ & $31+/-5.7$ \\
Systolic blood pressure, mmHg & $136.3+/-14.1$ \\
Diastolic blood pressure, mmHg & $81.9+/-7.9$ \\
HbA1c, \% ${ }^{\text {a }}$ & $7.1+/-0.9$ \\
History of hypertension, $n(\%)$ & $9(75)$ \\
History of diabetes, $n(\%)$ & $9(75)$ \\
\hline
\end{tabular}

Mean +/-SD unless otherwise indicated

${ }^{a}$ Data available in 7 patients text messages bank, cloud-based portal) for a future full-scale study.

\section{Principal findings}

This pilot study found that in patients with cardiometabolic disease, defined as type 2 diabetes and/or hypertension, the concomitant application of wireless BP monitoring and semi-personalized text messages based on the behavioral economic principles of automated hovering and nudging was feasible and acceptable. In patients with a wide age range and broad educational backgrounds, this pilot study demonstrates the feasibility and acceptability of a practical intervention strategy that capitalizes on the availability of simple wireless technology to implement the emerging science of behavioral economics to guide patient-tailored behavior change.

Results from a U.S. survey reported that the majority of people across all income groups possess a mobile phone [18] and text messages are low cost and can be easily automated. Recent evidence supports the effectiveness of mobile phone text message-based interventions to influence lifestyle and health-related behaviors, such as exercise [19], weight loss, and medication adherence [20], in patients with chronic diseases. In our pilot study, participants reported an increase in medication adherence at the end of the study.

When compared to usual care, text messaging interventions [21] and BP monitoring [22] alone showed a small effect on BP, whereas a greater reduction in BP was observed after a combined intervention of BP monitoring and lifestyle counseling [23]. In our pilot study, we observed an improvement of mean arterial BP after a combined intervention of PTMs and wireless BP home 


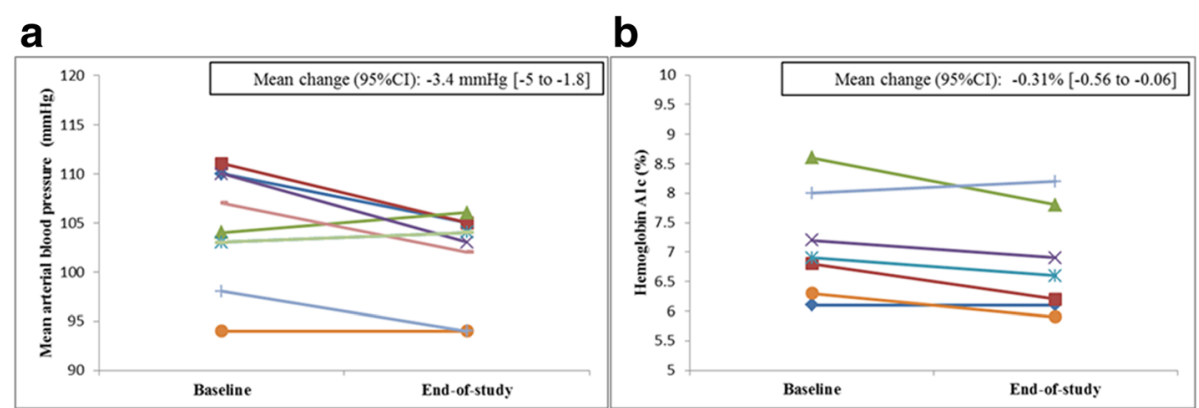

Fig. 2 a Mean arterial blood pressure in patients who received wireless blood pressure home monitoring. Data collected automatically via the monitors. b Hemoglobin A1c ascertained from available data in electronic health records collected during routine clinical care

monitoring, supporting the hypothesis of a positive synergic effect of such a concomitant intervention.

Our results are consistent with those of a RCT conducted among patients with coronary heart disease [24] where there was a greater improvement of systolic BP, BMI, physical activity and smoking status after a 6-month intervention of lifestyle-focused semi-personalized text messages [24].

In seven patients with available data, we observed a reduction of the HbA1c at the end of the study. This finding is in accordance with results from a recent RCT conducted among Hispanic patients with type 2 diabetes reporting a significant reduction of the HbA1c after a 6-month text message-based interventions using motivational, educational, and/or call-to-action text messages [25].

\section{Limitations}

The study's limitations (small number of patients and the lack of a control group) are consistent with the pilot nature of the study, whose main goal was to evaluate feasibility and acceptability and refine critical aspects of the overall approach [26]. Another limitation was that it was unclear to the investigators whether patients were receiving the text messages; therefore, a future study would send a bi-directional text at baseline asking patients to respond with a "yes" to indicate receipt. We also found that some older patients required additional training with the use of the BP home monitoring device.

\section{Conclusions}

Although behavioral economics and wireless technology have separately shown promise for improving care of chronic conditions in the short term, the concurrent application of strategies identified by the field of behavioral economics (e.g., "automated hovering", "nudging") and tools from wireless technology (e.g., monitoring, texting) has not been tried previously in patients with type 2 diabetes and/or hypertension in a real-world health care setting. Our pilot study showed that this approach is feasible and acceptable to patients and we expect that such a multi-component intervention to have synergistic benefits; however, larger studies are needed to confirm effectiveness.

\section{Abbreviations}

BP: Blood pressure; HbA1c: Hemoglobin A1c; IRB: Institutional review board; PTMs: Personalized text messages

\section{Acknowledgements}

The authors thank the participants for time and contributions.

\section{Funding}

This pilot study did not receive support from specific funding agencies in the public, commercial, or not-for-profit sectors.

\section{Availability of data and materials}

The datasets used and/or analyzed during the current study are available from the corresponding author on reasonable request.

\section{Authors' contributions}

EA, JBW, and AGP contributed to the concept and design of the study. All authors contributed to writing and critical revision of the manuscript. EA is the guarantor of this work and, as such, takes responsibility for the integrity and accuracy of the study design. All authors read and approved the final manuscript.

Ethics approval and consent to participate Ethical approval was granted by the Tufts IRB and all participants provided written informed consent.

Competing interests

The authors declare that they have no competing interests.

\section{Publisher's Note}

Springer Nature remains neutral with regard to jurisdictional claims in published maps and institutional affiliations.

\section{Author details}

'Division of Endocrinology, Diabetes and Metabolism, Tufts Medical Center, 800 Washington Street, \#268, Boston, MA 02111, USA. ²Division of Clinical Decision Making, Tufts Medical Center, 800 Washington Street, \#302, Boston, MA 02111, USA. ${ }^{3}$ Tufts University School of Medicine, 145 Harrison Ave, Boston, MA 02111, USA. ${ }^{4}$ Northeastern University, 360 Huntington Ave, Boston, MA 02115, USA. 
Received: 10 September 2018 Accepted: 4 January 2019

Published online: 15 January 2019

\section{References}

1. Asch DA, Muller RW, Volpp KG. Automated hovering in health care-watching over the 5000 hours. N Engl J Med. 2012;367(1):1-3.

2. Egan BM, Li J, Qanungo S, Wolfman TE. Blood pressure and cholesterol control in hypertensive hypercholesterolemic patients: national health and nutrition examination surveys 1988-2010. Circulation. 2013;128(1): 29-41.

3. Selvin E, Parrinello CM, Sacks DB, Coresh J. Trends in prevalence and control of diabetes in the United States, 1988-1994 and 1999-2010. Ann Intern Med. 2014;160(8):517-25

4. Lebeau JP, Cadwallader JS, Aubin-Auger I, Mercier A, Pasquet T, Rusch E, et al. The concept and definition of therapeutic inertia in hypertension in primary care: a qualitative systematic review. BMC Fam Pract. 2014;15:130.

5. Lee WC, Balu S, Cobden D, Joshi AV, Pashos CL. Prevalence and economic consequences of medication adherence in diabetes: a systematic literature review. Manag Care Interface. 2006;19(7):31-41.

6. Schroeder SA. Shattuck Lecture. We can do better-improving the health of the American people. N Engl J Med. 2007;357(12):1221-8.

7. Klonoff DC. The current status of mHealth for diabetes: will it be the next big thing? J Diabetes Sci Technol. 2013;7(3):749-58.

8. Steinhubl SR, Muse ED, Topol EJ. Can mobile health technologies transform health care? JAMA. 2013;310(22):2395-6.

9. Kahneman D. Thinking fast and slow. New York: Farrar, Straus \& Giroux; 2011

10. Emanuel EJ, Ubel PA, Kessler JB, Meyer G, Muller RW, Navathe AS, et al. Using behavioral economics to design physician incentives that deliver high-value care. Ann Intern Med. 2016;164(2):114-9.

11. Loewenstein G, Asch DA, Friedman JY, Melichar LA, Volpp KG. Can behavioural economics make us healthier? BMJ. 2012;344:e3482.

12. Rice T. The behavioral economics of health and health care. Annu Rev Public Health. 2013;34:431-47.

13. Thorgeirsson T, Kawachi I. Behavioral economics: merging psychology and economics for lifestyle interventions. Am J Prev Med. 2013;44(2):185-9.

14. mHealth. New horizons for health through mobile technologies. 2011.

15. Volpp KG, Troxel AB, Mehta SJ, Norton L, Zhu J, Lim R, et al. Effect of electronic reminders, financial incentives, and social support on outcomes after myocardial infarction: the HeartStrong randomized clinical trial. JAMA Intern Med. 2017;177(8):1093-101.

16. Culig J, Leppee M. From Morisky to Hill-bone; self-reports scales for measuring adherence to medication. Coll Antropol. 2014;38(1):55-62.

17. Thakkar J, Kurup R, Laba TL, Santo K, Thiagalingam A, Rodgers A, et al. Mobile telephone text messaging for medication adherence in chronic disease: a meta-analysis. JAMA Intern Med. 2016;176:340.

18. Duggan M. Cell phone activities 2013 pew research center, Internet and technology. 2013. Available from: http:/www.pewinternet.org/2013/09/19/ cell-phone-activities-2013/.

19. Fanning J, Mullen SP, MCAuley E. Increasing physical activity with mobile devices: a meta-analysis. J Med Internet Res. 2012;14(6):e161.

20. Marquez Contreras E, de la Figuera von Wichmann M, Gil Guillen V, YllaCatala A, Figueras M, Balana M, et al. Effectiveness of an intervention to provide information to patients with hypertension as short text messages and reminders sent to their mobile phone (HTA-Alert). Aten Primaria. 2004 34(8):399-405.

21. Bobrow K, Farmer AJ, Springer D, Shanyinde M, Yu LM, Brennan T, et al. Mobile phone text messages to support treatment adherence in adults with high blood pressure (SMS-text adherence support [StAR]): a single-blind, randomized trial. Circulation. 2016;133(6):592-600.

22. Wetzels GE, Nelemans PJ, Schouten JS, Dirksen CD, van der Weijden $T$, Stoffers $\mathrm{HE}$, et al. Electronic monitoring of adherence as a tool to improve blood pressure control. A randomized controlled trial. Am J Hypertens. 2007;20(2):119-25.

23. Tucker KL, Sheppard JP, Stevens R, Bosworth HB, Bove A, Bray EP, et al. Selfmonitoring of blood pressure in hypertension: a systematic review and individual patient data meta-analysis. PLoS Med. 2017;14(9):e1002389.
24. Chow CK, Redfern J, Hillis GS, Thakkar J, Santo K, Hackett ML, et al. Effect of lifestyle-focused text messaging on risk factor modification in patients with coronary heart disease: a randomized clinical trial. JAMA. 2015; 314(12):1255-63.

25. Fortmann AL, Gallo LC, Garcia MI, Taleb M, Euyoque JA, Clark T, et al. Dulce digital: an mHealth SMS-based intervention improves glycemic control in hispanics with type 2 diabetes. Diabetes Care. 2017;40(10):1349-55.

26. Kistin C, Silverstein M. Pilot studies: a critical but potentially misused component of interventional research. JAMA. 2015;314(15):1561-2.

\section{Ready to submit your research? Choose BMC and benefit from:}

- fast, convenient online submission

- thorough peer review by experienced researchers in your field

- rapid publication on acceptance

- support for research data, including large and complex data types

- gold Open Access which fosters wider collaboration and increased citations

- maximum visibility for your research: over $100 \mathrm{M}$ website views per year

At BMC, research is always in progress.

Learn more biomedcentral.com/submissions 